\title{
Cifra de Hill: uma aplicação ao estudo de matrizes
}

\section{Hill Cipher: an application to the study of matrices}

\author{
Lucas Diego Antunes Barbosa \\ Instituto Federal de Educação, Ciência e Tecnologia do Norte de MG - IFNMG, Salinas, \\ MG \\ lucas.barbosa@ifnmg.edu.br \\ Mariana Garabini Cornelissen \\ Universidade Federal de São João del Rei - UFSJ, Ouro Branco, MG \\ mariana@ufsj.edu.br
}

Resumo: O aprendizado de um conteúdo matemático pode se tornar mais atrativo para o estudante se estiver associado a alguma aplicação. Partindo desse princípio, esse trabalho apresenta aos professores de matemática do Ensino Médio uma proposta de aplicação do conteúdo de matrizes à uma técnica criptográfica, ou seja, uma técnica para codificar e decodificar mensagens, chamada Cifra de Hill. Além de mostrar uma aplicação de um conteúdo matemático, esse trabalho também apresenta uma possibilidade de trabalhar a integração de diferentes disciplinas em sala de aula, já que fazemos uso de conhecimentos básicos de programação de computadores, conteúdo essencial para os estudantes no dia de hoje, na proposta de aula aqui apresentada.

Palavras-chave: criptografia; matrizes; Hill; cifra.

Abstract: Learning mathematical content may become more attractive to students if they are associated with an application. Based on this principle, this work presents to teachers of high school mathematics a proposal to apply matrix content to a cryptographic technique, that is, a technique for encoding and decoding messages, called Hill Cipher. In addition to showing an application of mathematical content, this work also presents a possibility of working the integration of different disciplines in the classroom, since we make use of basic knowledge of computer programming, essential content for students today, in the lesson proposal presented here.

Key-words: encryption; matrices; Hill; cipher.

\section{Introdução}

O mundo está sofrendo várias transformações, mas alguns aspectos permanecem iguais. Hoje, assim como no passado, para muitos jovens, aprender matemática na escola é uma experiência difícil e, às vezes, desestimulante. Segundo D'Ambrosio [1] os professores em geral mostram a matemática como um corpo de conhecimentos acabado e polido. "Ao aluno não é dado em nenhum momento a oportunidade ou gerada a necessidade de criar nada, nem mesmo uma solução mais interessante. O aluno assim, passa a acreditar que na aula de Matemática o seu papel é passivo e desinteressante"[1].

Recebido em 30/08/2017 - Aceito em 20/11/2017

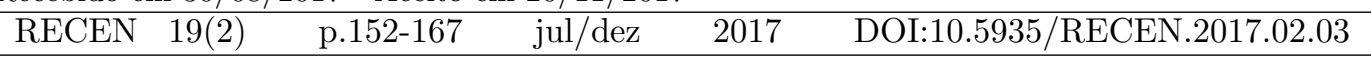


Aprender não é a simples aquisição de técnicas e habilidades e nem a memorização de algumas explicações e teorias [2]. Por isso, sempre que possível, sugere-se que o professor mostre uma aplicação com o objetivo de estimular e facilitar o aprendizado de um determinado conteúdo. "A possibilidade de compreender conceitos e procedimentos matemáticos é necessária tanto para tirar conclusões e fazer argumentações, quanto para o cidadão agir como consumidor prudente ou tomar decisões em sua vida pessoal e profissional" [3]. Nesta mesma direção, Charlot [4] aponta que a educação não consiste em transmitir conhecimentos acabados, mas em propor aos alunos situações e problemas que desencadeiam uma atividade intelectual que, com a ajuda do professor, leve ao conhecimento. E ainda, que essas situações provoquem o aluno a despertar algum conhecimento anterior, no contexto da aprendizagem significativa. A aprendizagem significativa, conforme Moreira [5], é aquela em que as ideias expressas simbolicamente interagem de maneira substantiva e não arbitrária com aquilo que o aluno já sabe.

Partindo dessa teoria de aprendizagem, esse trabalho tem o objetivo de apresentar aos professores de matemática uma proposta de aplicação do conteúdo de matrizes à uma técnica de criptografia, ou seja, um procedimento para codificar e decodificar mensagens. Atualmente, existem diversos trabalhos já publicados sobre esse tema. Abaixo escolhemos três pesquisas que mais se aproximam do nosso estudo.

O trabalho de mestrado intitulado "Criptografia matricial aplicada ao ensino médio", Schurman [6] teve como objetivo geral apresentar uma sequência didática para trabalhar o conteúdo matrizes com alunos do ensino médio. O autor aponta que o conceito de matrizes é amplamente utilizado em diversas áreas do conhecimento, tais como Economia, Engenharia, Tecnologia, dentre outros. E ainda mais importante que entender o conceito de matrizes é "preciso atribuir sentindo a ele, pois com isso os alunos poderão perceber a necessidade de representação matricial nos diversos fenômenos que os cercam" [6].

Já na dissertação intitulada "Criptografia: uma engenharia didática com funções matrizes e cifra de Hill para o ensino médio", Marinho [7] propôs a implementação de uma engenharia didática para o tratamento do tema criptografia, associado aos conteúdos de matemática trabalhados no ensino médio. O pesquisador afirma que "atividades envolvendo o tema criptografia abrem caminho para a introdução, em sala de aula, de tecnologias da informação e comunicação" [7].

A pesquisa intitulada "Uma proposta para ampliar a perspectiva de professores e alunos em relação ao estudo de matrizes", teve como objetivo proporcionar a professores da rede estadual de São Paulo e alunos de licenciatura em matemática, uma alternativa eficiente e significativa para o estudo de matrizes com foco em transformação geométricas. Neste estudo, Oliveira [8] aponta que a matemática sem dúvida alguma está entre as disciplinas mais rejeitadas por alunos do ensino regular. No entanto, provavelmente na maioria dos casos essa rejeição está associada a abordagens inadequadas de conteúdos, as quais não permitem que os alunos percebam as possíveis aplicações e transformações dos conhecimentos estudados em ferramentas utilizadas por toda sociedade.

No caso desse trabalho, a técnica criptográfica escolhida é a chamada Cifra de Hill em homenagem ao seu criador, o americano Lester S. Hill, que desenvolveu essa técnica em 1929. A justificativa para esta escolha são as possibilidades de abordar diversos conteúdos matemáticos com esse método, como será visto adiante. Conforme Conceição [9] a criptografia, por fazer parte do cotidiano dos alunos, se torna um método muito eficaz para o desenvolvimento de conceitos matemáticos, não somente no Ensino Médio, como também no Ensino Fundamental e a partir daí, torna-se um motivador para a aprendizagem da matemática.

Dessa forma, esse trabalho apresenta uma aplicação de um conteúdo matemático que 
Revista Ciências Exatas e Naturais, Vol.19, nº.2, Jul/Dez, 2017

permite trabalhar os conteúdos de matrizes, determinantes, máximo divisor comum, noções de aritmética modular, criptografia e programação de computadores em sala de aula, mostrando assim uma possível relação entre teoria e prática matemática. Espera-se que esse trabalho instigue a curiosidade dos professores e dos alunos na relação de teoria e prática matemática, com o intuito de melhorar a relação de ensino e de aprendizagem.

\section{Criptografia}

Desde o surgimento da humanidade existe a necessidade de se obter maior segurança na transmissão de informações que são enviadas por diversos meios de comunicação. Em tempos passados de guerra se observou o uso de técnicas especiais para o envio de mensagens secretas para as tropas, como foi o caso de Júlio César, imperador de Roma, quando suas tropas estavam pela Europa em guerra [10]. O imperador deslocava o alfabeto três casas adiante para codificar suas mensagens, como pode ser visualizado pela tabela abaixo.

Tabela 1. Cifra de César

\begin{tabular}{c}
\hline letra $\rightarrow$ letra correspondente \\
\hline \hline $\mathrm{A} \rightarrow \mathrm{D}$ \\
$\mathrm{B} \rightarrow \mathrm{E}$ \\
\hline $\mathrm{C} \rightarrow \mathrm{F}$ \\
\hline $\mathrm{D} \rightarrow \mathrm{G}$ \\
\hline $\mathrm{E} \rightarrow \mathrm{H}$ \\
\hline $\mathrm{F} \rightarrow \mathrm{I}$ \\
\hline $\mathrm{e}$ assim por diante
\end{tabular}

Neste caso, se a mensagem a ser enviada fosse: ACABEM COM O INIMIGO, as tropas receberiam a seguinte mensagem cifrada DFDEHPFRPRNQNPNLR. De acordo com a pesquisa realizada por Fiarresga [11], o relato mais grotesco de transmissão de mensagens codificadas foi usado por Histieu ao transmitir uma mensagem a Aristágoras de Mileto. Histieu raspou a cabeça de um indivíduo, escreveu no seu couro cabeludo a mensagem que queria enviar, esperou que o cabelo do indivíduo voltasse a crescer e enviou-o em viagem até Aristágoras. O indivíduo quando chegou, raspou novamente a cabeça e mostrou a mensagem à Aristágoras de Mileto.

Essa transmissão de informações de forma oculta é conhecida por criptografia. Portanto, criptografia é a técnica de esconder uma escrita e o seu significado é de origem grega (kripto = escondido, oculto e grápho = grafia, escrita). Segundo Evaristo e Perdigão [12] a criptografia pode ser entendida como a ação de reescrever um texto de modo que apenas as pessoas autorizadas pelo autor do texto sejam capazes de compreendê-lo. O texto normalmente é chamado de mensagem, uma pessoa autorizada a ler a mensagem é chamada destinatário e o autor da mensagem é chamado de remetente. Chamamos de chave criptográfica algo necessário para a codificação ou decodificação da mensagem. Dessa forma, podemos dizer que a criptografia estuda os métodos para codificar uma mensagem de modo que só o seu destinatário legítimo consiga interpretá-la.

Uma vez que a comunicação entre as pessoas é inevitável no atual momento de grande avanço tecnológico, percebemos o quão sério é a transmissão de informações sigilosas. Por 
exemplo, uma instituição financeira possui informações particulares e importantes de muitas pessoas e tais informações podem ser acessadas caso não haja um sistema de segurança eficaz. Informações secretas como senhas podem ser interceptadas por pessoas inescrupulosas e utilizadas de forma prejudicial aos proprietários, causando grande prejuízo a estes, pois, com a senha de uma conta bancária, podem ser realizados saques e empréstimos em favor de terceiros. Além do prejuízo com contas bancárias, como no exemplo das instituições financeiras, podemos também citar como exemplo a possibilidade de quebra de sigilo de emails, tratando-se de informações compartilhadas via internet, caso um sistema criptográfico não seja utilizado. A quebra do sigilo de e-mails pode provocar grandes danos, como a descoberta de endereço, telefone, local de trabalho, o que pode ser perigoso quando dados tão particulares forem parar na mão de pessoas maldosas. Neste sentido, a criptografia assume um importante papel.

Existem dois tipos de criptografia: simétrica e assimétrica. Na criptografia simétrica, a forma de codificar e decodificar uma mensagem é a mesma. Já na criptografia assimétrica a maneira de codificar não é a mesma de decodificar. No caso da criptografia assimétrica, a chave de codificação é pública e no caso da criptografia simétrica essa chave é privada. São exemplos de métodos criptográficos simétricos: Cifra de César, Cifra de Hill, Data Encryption Standard (DES)e Advanced Encryption Standard (AES). Como método criptográfico assimétrico, podemos citar o método mais famoso e utilizado hoje em dia, que é o método RSA, desenvolvido em 1978 por Ronald Rivest, Adi Shamir e Leonard Adleman (daí o nome RSA), pesquisadores na época do Instituto de Tecnologia de Massachusetts. Mais adiante, falaremos um pouco sobre cada um desses métodos.

De acordo com Coutinho [13] estes códigos foram criados para o uso em aplicações comerciais, e não na comunicação entre espiões. Por isso, os códigos modernos são todos de chave pública. Esta é uma ideia introduzida em 1976 por W. Diffie e M.E. Hellman da Universidade da Califórnia. Em um código de chave pública saber codificar não implica saber decodificar! Isto parece impossível: se sei codificar, para decodificar basta desfazer o que fiz. Desfazer o processo de codificação pode não ser tão simples quanto parece.

O DES é um método criptográfico que foi designado, pelos Estados Unidos da América, como algoritmo padrão de criptografia em 1977 e foi amplamente utilizado internacionalmente. É um algoritmo de cifra em blocos, isto é, tal algoritmo opera sobre agrupamentos de tamanho fixo, chamado de blocos. Atualmente, o DES sozinho é considerado inseguro para muitas aplicações e, em 1997, foi substituído pelo AES, mas ainda continuou a ser utilizado em larga escala até 2004 com algumas modificações. O AES tornou-se o algoritmo padrão em 2002 e em 2006 já era um dos algoritmos mais populares utilizados para criptografia simétrica. O AES também é um algoritmo de cifra em bloco, porém com um tamanho de chave maior que o DES. Uma descrição detalhada de tais algoritmos pode ser encontrada em [10]. A criptografia RSA é um método de chave pública cuja codificação baseia-se em um determinado número que é o produto de dois números primos e a decodificação por esse método depende da fatoração desse número, ou seja, dos números primos que o originou. Portanto, sua segurança é garantida com a escolha de um par de números primos grandes. Ao leitor interessado nesse método criptográfico recomenda-se o livro [13]. A cifra de Hill, que é uma técnica de criptografia simétrica, com chave privada, será o assunto de uma próxima seção. Antes, será necessário revisar alguns conceitos e resultados sobre matrizes e aritmética modular. 
Revista Ciências Exatas e Naturais, Vol.19, nº.2, Jul/Dez, 2017

\section{Preliminares}

Apresentamos nesta seção algumas definições e resultados sobre matrizes e também sobre aritmética modular que serão necessários para o entendimento do método de Hill. Uma leitura mais detalhada sobre esses conteúdos pode ser encontrada em [14] e [15].

Definição 3.1 Seja $k \in \mathbb{N}-\{0\}$. Dizemos que dois números inteiros a e b são congruentes módulo $k$ se os restos da divisão euclidiana de a e b por $k$ são iguais. Neste caso, escrevemos $a \equiv b \bmod k$.

Pode-se mostrar que $\equiv$ é uma relação de equivalência em $\mathbb{Z}$ cuja classe de equivalência módulo $k$ de $a \in \mathbb{Z}$ é dada por

$$
[a]=\{x \in \mathbb{Z} ; x \equiv a \bmod k\}=\{x=a+k q \mid q \in \mathbb{Z}\}
$$

Além disso, para cada $a \in \mathbb{Z}$ existe um e somente um $r \in \mathbb{Z}, 0 \leq r<k$, tal que $[a]=[r]$. Logo, existem exatamente $k$ classes de equivalência módulo $k$ distintas:

$$
[0],[1],[2], \ldots,[k-1]
$$

O conjunto de todas essas classes módulo $k$ será representado por $\mathbb{Z}_{k}$. O leitor interessado pode consultar esse conteúdo em [16].

Definição 3.2 Um elemento $[a] \in \mathbb{Z}_{k}$ será dito invertível, quando existir $[b] \in \mathbb{Z}_{k}$ tal que $[a][b]=[1]$. O elemento $[b] \in \mathbb{Z}_{k}$ é único (ver demonstração em [17]]) e é dito o inverso de $[a]$.

Proposição $3.1[a] \in \mathbb{Z}_{k}$ é invertivel se, e somente se, $m d c(a, k)=1$

Demonstração: Suponha que $[a] \in \mathbb{Z}_{k}$ é invertivel, logo, existe $[b] \in \mathbb{Z}_{k}$ tal que:

$$
[a] .[b]=[1] \Leftrightarrow a . b \equiv 1 \bmod k \Leftrightarrow a . b-1 \equiv 0 \bmod k
$$

o que implica que existe $q \in \mathbb{Z}$ tal que a.b-1 = q.k. Logo, a.b-q.k=1 donde $m d c(a, k)=1$.

Suponha agora que $m d c(a, k)=1$. Então existem $q, b \in \mathbb{Z}$ tais que:

$$
a . b+q . k=1 .
$$

Portanto, $a . b \equiv 1 \bmod k$, ou seja, $[a][b]=[1]$, como queríamos demonstrar.

Veremos agora como encontrar o inverso de um número em $\mathbb{Z}_{k}$.

Suponha $a \in \mathbb{Z}_{k}$ invertível e seja $a^{-1} \in \mathbb{Z}_{k}$ o seu inverso; então $\operatorname{mdc}(a, k)=1$, o que implica que existem $b, q \in \mathbb{Z}$ tais que:

$$
1=a \cdot b+k \cdot q
$$

donde

$$
1 \equiv a . b \bmod k
$$

Multiplicando os dois membros da igualdade por $a^{-1}$, obtemos: 


$$
a^{-1} \equiv b \bmod k
$$

Precisamos portanto encontrar $b$. Os valores de $b$ e $q$ podem ser encontrados utilizando o algoritmo de Euclides, como pode ser visto no exemplo abaixo.

Exemplo 3.1 Neste exemplo iremos encontrar o inverso de 55 em $\mathbb{Z}_{26}$. Como $m d c(55,26)=$ 1 , sabemos que 55 é invertivel em $\mathbb{Z}_{26}$ e, além disso, existem $b, q \in \mathbb{Z}$ tais que

$$
55 . b+26 . q=1
$$

Para encontrarmos b que, conforme dito anteriormente, será o inverso de 55 em $\mathbb{Z}_{26}$, devemos inicialmente dividir 55 por 26, através da divisão euclidiana, obtendo um quociente e um resto:

$$
55=26.2+3
$$

Agora, devemos dividir 26 pelo resto encontrado, no caso 3, e continuar esse processo, ou seja, de dividir o dividendo pelo resto, até encontrarmos resto nulo.

$$
\begin{aligned}
& 26=3.8+2 \\
& 3=2.1+1 \\
& 2=1.2+0
\end{aligned}
$$

Observe que esse é o processo que utilizamos para determinar o máximo divisor comum $(m d c)$ entre dois números. O último resto não nulo encontrado é o mdc entre 55 e 26. Agora, partindo da última equação cujo resto é não nulo, e utilizando as equações acima, conseguimos encontrar $b$ e q. Vejamos:

$$
1=3-2.1
$$

Mas, das equações anteriores, temos que $2=26-3.8$ e $3=55-26.2$, donde

$$
\begin{aligned}
& 1=3-2.1=3-(26-3.8) .1=3-26.1+3.8 .1=3.9-26.1 \\
& 1=(55-26.2) .9-26.1=55.9-26.2 .9-26.1=55.9-26.19
\end{aligned}
$$

donde

$$
1=55.9-26.19
$$

o que implica que 9 é o inverso de 55 em $\mathbb{Z}_{26}$.

Definição 3.3 Dada uma matriz quadrada $A$ de ordem $n$, chama-se de inversa de $A$ à matriz quadrada $B$ de ordem $n$ tal que:

$$
A \cdot B=B \cdot A=I_{n}
$$

onde $I_{n}$ é a matriz identidade de ordem n. Se uma matriz A possui inversa, então sua inversa é única (ver demonstração em [14]) e será denotada por $A^{-1}$. 
Revista Ciências Exatas e Naturais, Vol.19, nº.2, Jul/Dez, 2017

Definição 3.4 Seja $A=\left(a_{i j}\right)_{n \times n}$ uma matriz quadrada de ordem $n$. O determinante $d a$ matriz $A$, denotado por $\operatorname{det}(A)$, é o número real dado por:

$$
\operatorname{det}(A)=\sum_{i=1}^{n} a_{i j} \cdot(-1)^{i+j} \operatorname{det}(A(i \mid j))
$$

onde $j$ é qualquer inteiro fixo entre 1 e n e $A(i \mid j)$ é a matriz formada a partir da matriz $A$ suprimindo sua i-ésima linha e sua j-ésima coluna.

Sabemos que uma matriz é invertível se, e somente se, seu determinante é não nulo. Uma demonstração desse fato pode ser encontrada em [18].

Definição 3.5 Define-se o cofator do elemento $a_{i j}$ da matriz $A$ como

$$
\Delta_{i j}(A)=(-1)^{i+j} \operatorname{det}(A(i \mid j)) .
$$

A matriz $B=\left(\Delta_{i j}(A)\right)_{n \times n}$ será chamada de matriz dos cofatores da matriz $A$ e sua transposta será chamada de matriz adjunta de $A$ e denotada por adj $(A)$.

Lema 3.1 Se A é uma matriz quadrada de ordem n, então

$$
\begin{aligned}
& a_{k 1} \Delta_{i 1}+a_{k 2} \Delta_{i 2}+\ldots+a_{k n} \Delta_{i n}=0 \text { se } k \neq i \\
& a_{1 k} \Delta_{1 j}+a_{2 k} \Delta_{2 j}+\ldots+a_{n k} \Delta_{n j}=0 \text { se } k \neq j
\end{aligned}
$$

para $i, j=1, \ldots, n$.

Demonstração: Definimos a matriz $A^{\prime}$ como sendo a matriz obtida de $A$ substituindo a i-ésima linha de $A$ por sua k-ésima linha, ou seja,

$$
A=\left(\begin{array}{c}
A_{1} \\
\vdots \\
A_{i} \\
\vdots \\
A_{k} \\
\vdots \\
A_{n}
\end{array}\right) \text { e } A^{\prime}=\left(\begin{array}{c}
A_{1} \\
\vdots \\
A_{k} \\
\vdots \\
A_{k} \\
\vdots \\
A_{n}
\end{array}\right)
$$

Como a matriz $A^{\prime}$ possui duas linhas iguais, logo det $A^{\prime}=0$. O desenvolvimento do determinante de $A^{\prime}$ segundo a i-ésima linha é exatamente a equação 1 . De modo análogo prova-se a equação 2 , ainda usando o fato que $\operatorname{det}(A)=\operatorname{det}\left(A^{t}\right)$ onde $A^{t}$ é matriz transposta de $A$.

Proposição 3.2 Seja A um matriz quadrada de ordem n. Então:

$$
\operatorname{adj}(A) \cdot A=\operatorname{det}(A) \cdot I_{n}
$$

Demonstração: $O$ produto da matriz adjunta de A pela matriz A é dado por: 


$$
\left(\begin{array}{ccc}
\Delta_{11} & \cdots & \Delta_{n 1} \\
\vdots & \vdots & \vdots \\
\Delta_{1 i} & \cdots & \Delta_{n i} \\
\vdots & \vdots & \vdots \\
\Delta_{1 n} & \cdots & \Delta_{n n}
\end{array}\right) \cdot\left(\begin{array}{ccccc}
a_{11} & \cdots & a_{1 j} & \cdots & a_{1 n} \\
\vdots & \vdots & \vdots & \vdots & \vdots \\
a_{i 1} & \cdots & a_{i j} & \cdots & a_{i n} \\
\vdots & \vdots & \vdots & \vdots & \vdots \\
a_{n 1} & \cdots & a_{n j} & \cdots & a_{n n}
\end{array}\right)
$$

O elemento da posição $i, j$ de $\operatorname{adj}(A) . A$ é

$$
(a d j(A) . A)_{i j}=\sum_{k=1}^{n} a_{i k} \Delta_{j k}=a_{i 1} \Delta_{j 1}+a_{i 2} \Delta_{j 2}+\ldots+a_{i n} \Delta_{j n}
$$

Pelo lema 3.1, equação 2, e pela definição 3.4 temos que:

$$
(\operatorname{adj}(A) \cdot A)_{i j}=\left\{\begin{array}{c}
\operatorname{det}(A), \text { se } i=j \\
0, \text { se } i \neq j
\end{array}\right.
$$

Assim,

$$
\operatorname{adj}(A) \cdot A=\left(\begin{array}{cccc}
\operatorname{det}(A) & 0 & \cdots & 0 \\
0 & \operatorname{det}(A) & \cdots & 0 \\
\vdots & \cdots & \cdots & \vdots \\
0 & 0 & \cdots & \operatorname{det}(A)
\end{array}\right)=\operatorname{det}(A) \cdot I_{n}
$$

Proposição 3.3 Se A é uma matriz invertível, então

$$
A^{-1}=\frac{1}{\operatorname{det} A} \cdot \operatorname{adj}(A)
$$

Demonstração: Se $A$ é invertível então $\operatorname{det}(A) \neq 0$. Logo, segue pela proposição 3.2 que

$$
\left(\frac{1}{\operatorname{det}(A)} \operatorname{adj}(A)\right) \cdot A=I_{n}
$$

donde $A^{-1}=\frac{1}{\operatorname{det}(A)} \operatorname{adj}(A)$

Corolário 3.1 Dada uma matriz $A=\left(a_{i j}\right)_{n \times n}$, o determinante da matriz A será invertível em $\mathbb{Z}_{k}$ se o $m d c(\operatorname{det} A, k)=1$.

A demonstração desse corolário é direta pelo que foi visto na proposição 3.1. Com isso, estamos prontos para entender o método de Hill. 
Revista Ciências Exatas e Naturais, Vol.19, nº.2, Jul/Dez, 2017

\section{Cifra de Hill}

A cifra de Hill faz parte da época das cifras com papel e lápis, ele é inseguro contra ataque via computador e pode ser decodificado facilmente. Abaixo, segue um roteiro detalhando passo a passo desse algoritmo de codificação e decodificação, assim como um exemplo.

1. Inicialmente devemos converter as letras da mensagem a ser criptografada em números. Isso pode ser feito de diversas maneiras, dependendo de qual número será associado a cada letra. Aqui neste trabalho, usaremos a tabela ASCII (American Standard Code for Interchange Information) na base decimal para essa conversão, que é a tabela mais utilizada na área computacional. Sem perda de generalidade, podemos trabalhar somente com as letras maiúsculas.

Tabela 2. Conversão das letras maiúsculas para o sistema decimal, conforme a tabela ASCII

\begin{tabular}{ccccccccccccc}
\hline $\mathrm{A}$ & $\mathrm{B}$ & $\mathrm{C}$ & $\mathrm{D}$ & $\mathrm{E}$ & $\mathrm{F}$ & $\mathrm{G}$ & $\mathrm{H}$ & $\mathrm{I}$ & $\mathrm{J}$ & $\mathrm{K}$ & $\mathrm{L}$ & $\mathrm{M}$ \\
\hline 65 & 66 & 67 & 68 & 69 & 70 & 71 & 72 & 73 & 74 & 75 & 76 & 77 \\
\hline $\mathrm{N}$ & $\mathrm{O}$ & $\mathrm{P}$ & $\mathrm{Q}$ & $\mathrm{R}$ & $\mathrm{S}$ & $\mathrm{T}$ & $\mathrm{U}$ & $\mathrm{V}$ & $\mathrm{W}$ & $\mathrm{X}$ & $\mathrm{Y}$ & $\mathrm{Z}$ \\
\hline 78 & 79 & 80 & 81 & 82 & 83 & 84 & 85 & 86 & 87 & 88 & 89 & 90 \\
\hline
\end{tabular}

2. Agora devemos agrupar a sequência numérica obtida em vetores coluna de tamanho $n$ onde $n$ pode ser qualquer número natural não nulo. Caso o último vetor tenha tamanho menor que $n$, repita o último número do vetor até completar o tamanho $n$.

3. O terceiro passo é escolher uma matriz $A=\left(a_{i j}\right)_{n \times n}$ que será a chave de codificação. O determinante da matriz $A$ deve ser invertível em $\mathbb{Z}_{k}$, isto é, de acordo com o corolário 3.1 , devemos escolher $A$ de forma que $\operatorname{mdc}(\operatorname{det} A, k)=1$. Aqui $k$ é o número de símbolos possíveis de acordo com a tabela utilizada. No nosso caso, que estamos trabalhando só com as letras maiúsculas, $k=26$.

4. Em seguida, iniciamos a codificação que consiste simplesmente em multiplicar à esquerda a matriz chave $A$ por uma matriz $B$ cujas colunas são formadas pelos vetores coluna do passo 2 com cada entrada subtraída de 65 . Deve-se subtrair o número 65 , pois assim tem-se a classe residual módulo 26.

$$
A . B=\left(\begin{array}{cccc}
a_{11} & a_{12} & \cdots & a_{n 1} \\
a_{21} & a_{22} & \cdots & a_{n 2} \\
\vdots & \vdots & \ddots & \vdots \\
a_{n 1} & a_{n 2} & \cdots & a_{n n}
\end{array}\right) \cdot\left(\begin{array}{ccc}
b_{11} & \cdots & b_{n 1} \\
b_{21} & \cdots & b_{n 2} \\
\vdots & \ddots & \vdots \\
b_{n 1} & \cdots & b_{n m}
\end{array}\right)
$$

5. Após efetuar o produto das matrizes, caso alguma entrada da matriz final seja maior ou igual a 26, devemos trocar esse número pelo seu resto na divisão por 26.

6. Finalmente some 65 a cada uma das entradas dessa matriz obtida e transforme seus vetores coluna em letras de acordo com a tabela 2. Essa mensagem formada é a codificação da mensagem original.

Faremos agora um exemplo desse algoritmo de codificação. Como estamos utilizando a tabela 2, só utilizamos as letras maiúsculas sem os espaços e os acentos. 
BARBOSA, L. D. A. e CORNELISSEN, M. G.

\section{Exemplo 1}

Mensagem original: CIFRADEHILL

Passo 1: Conversão da mensagem de acordo com a tabela 2

\begin{tabular}{ccccccccccc}
\hline $\mathrm{C}$ & $\mathrm{I}$ & $\mathrm{F}$ & $\mathrm{R}$ & $\mathrm{A}$ & $\mathrm{D}$ & $\mathrm{E}$ & $\mathrm{H}$ & $\mathrm{I}$ & $\mathrm{L}$ & $\mathrm{L}$ \\
\hline 67 & 73 & 70 & 82 & 65 & 68 & 69 & 72 & 73 & 76 & 76 \\
\hline
\end{tabular}

Passo 2: Agrupando a sequência numérica em vetores coluna de tamanho $n=3$ e repetindo o último número do vetor coluna:

$$
\left(\begin{array}{l}
67 \\
73 \\
70
\end{array}\right),\left(\begin{array}{l}
82 \\
65 \\
68
\end{array}\right),\left(\begin{array}{l}
69 \\
72 \\
73
\end{array}\right),\left(\begin{array}{l}
76 \\
76 \\
76
\end{array}\right)
$$

Passo 3: Escolha da chave de codificação. Neste caso, uma matriz $A$ de ordem 3 dada por

$$
A=\left(\begin{array}{ccc}
1 & 4 & 6 \\
0 & 1 & 5 \\
3 & -1 & 8
\end{array}\right)
$$

Observe que $\operatorname{det} \mathrm{A}=55$ e $\operatorname{mdc}(55,26)=1$.

Passo 4: Multiplicando a matriz A à esquerda pela matriz formada pelos vetores coluna com cada entrada subtraída de 65 , obtemos:

$$
\left(\begin{array}{ccc}
1 & 4 & 6 \\
0 & 1 & 5 \\
3 & -1 & 8
\end{array}\right) \cdot\left(\begin{array}{cccc}
2 & 17 & 4 & 11 \\
8 & 0 & 7 & 11 \\
5 & 3 & 8 & 11
\end{array}\right)=\left(\begin{array}{cccc}
64 & 35 & 80 & 121 \\
33 & 15 & 47 & 66 \\
38 & 75 & 69 & 110
\end{array}\right)
$$

Passos 5: Agora devemos determinar os restos da divisão euclidiana de cada entrada por 26, obtendo:

$$
\left(\begin{array}{cccc}
12 & 9 & 2 & 17 \\
7 & 15 & 21 & 14 \\
12 & 23 & 17 & 6
\end{array}\right)
$$

Passo 6: E finalmente, somando 65 a cada entrada da matriz anterior, chegamos à matriz abaixo

$$
\left(\begin{array}{llll}
77 & 74 & 67 & 82 \\
72 & 80 & 86 & 79 \\
77 & 88 & 82 & 71
\end{array}\right)
$$

Fazendo a conversão dos números obtidos em letras de acordo com a tabela 2 concluímos que o destinatário receberá a seguinte mensagem codificada: MHMJPXCVRROG

Agora, passaremos ao processo de decodificação. Segue abaixo um roteiro detalhando passo a passo o que deve ser feito para decodificar uma mensagem codificada utilizando o processo de Hill. 
Revista Ciências Exatas e Naturais, Vol.19, nº.2, Jul/Dez, 2017

1. Além da mensagem codificada, o remetente também deve enviar para o destinatário a matriz chave de codificação, $A=\left(a_{i j}\right)_{n \times n}$ utilizada. O destinatário ao receber a mensagem codificada precisará converter as letras em números conforme a tabela utilizada pelo remetente, subtrair 65 de cada um desses números e formar uma matriz $C=\left(c_{i j}\right)_{n \times m}$, ou seja, ele deve agrupar a sequência numérica obtida em vetores coluna de tamanho $n$.

2. Agora, o destinatário precisa determinar o inverso do determinante da matriz $A$ em $\mathbb{Z}_{26}$ e, em seguida, determinar a matriz adjunta de $A$.

3. O próximo passo é multiplicar o inverso do determinante em $\mathbb{Z}_{26}$ e a matriz adjunta de $A$ encontrados no passo anterior pela matriz C. Observe que aqui estamos simplesmente fazendo o produto $A^{-1}$.C.

4. Em seguida, encontre o resto da divisão euclidiana de cada uma das entradas dessa matriz por 26 .

5. E finalmente, some 65 a cada uma das entradas.

Dessa forma, o destinatário descobrirá a mensagem original enviada. Após a codificação e decodificação podemos perceber de fato que o método é inseguro. Pela codificação e por alguns cálculos matemáticos, a decodificação é feita de forma fácil.

Vamos aplicar esse algoritmo para recuperar a mensagem do exemplo 1.

\section{Exemplo 2}

Passo 1: Suponha que o destinatário recebeu a matriz chave de ordem 3 e a mensagem abaixo:

\section{MHMJPXCVRROG}

$$
A=\left(\begin{array}{ccc}
1 & 4 & 6 \\
0 & 1 & 5 \\
3 & -1 & 8
\end{array}\right)
$$

Tomando como referência a tabela 2, o destinatário pode transformar esse bloco de letras na seguinte matriz:

$$
B=\left(\begin{array}{llll}
77 & 74 & 67 & 82 \\
72 & 80 & 86 & 79 \\
77 & 88 & 82 & 71
\end{array}\right)
$$

Subtraindo 65 de cada entrada da matriz anterior obtém-se a matriz: 


$$
C=\left(\begin{array}{cccc}
12 & 9 & 2 & 17 \\
7 & 15 & 21 & 14 \\
12 & 23 & 17 & 6
\end{array}\right)
$$

Passo 2 : Como o determinante da matriz chave é 55 , precisamos encontrar o inverso de 55 em $\mathbb{Z}_{26}$. Pelo exemplo 3.1, temos que o inverso de 55 em $\mathbb{Z}_{26}$ é 9 .

Obtendo a matriz adjunta de A, tem-se a matriz:

$$
\operatorname{adj}(A)=\left(\begin{array}{ccc}
13 & -38 & 14 \\
15 & -10 & -5 \\
-3 & 13 & 1
\end{array}\right)
$$

Passo 3: O próximo passo agora é multiplicar 9.adj(A).C:

$$
\begin{aligned}
& 9 .\left(\begin{array}{ccc}
13 & -38 & 14 \\
15 & -10 & -5 \\
-3 & 13 & 1
\end{array}\right) \cdot\left(\begin{array}{cccc}
12 & 9 & 2 & 17 \\
7 & 15 & 21 & 14 \\
12 & 23 & 17 & 6
\end{array}\right) \\
= & \left(\begin{array}{ccc}
117 & -342 & 126 \\
135 & -90 & -45 \\
-27 & 117 & 9
\end{array}\right) \cdot\left(\begin{array}{cccc}
12 & 9 & 2 & 17 \\
7 & 15 & 21 & 14 \\
12 & 23 & 17 & 6
\end{array}\right) \\
= & \left(\begin{array}{cccc}
522 & -1179 & -4806 & -2043 \\
450 & -1170 & -2385 & 765 \\
603 & 1719 & 2556 & 1233
\end{array}\right)
\end{aligned}
$$

Passo 4: Em seguida, encontre o resto da divisão euclidiana de cada uma das entradas dessa matriz por 26

$$
=\left(\begin{array}{cccc}
2 & 17 & 4 & 11 \\
8 & 0 & 7 & 11 \\
5 & 3 & 8 & 11
\end{array}\right)
$$

Passo 5: Somando 65 a cada entrada da matriz acima e consultando a tabela 2, obtém-se a mensagem codificada.

$$
\begin{aligned}
& =\left(\begin{array}{llll}
67 & 82 & 69 & 76 \\
73 & 65 & 72 & 76 \\
70 & 68 & 73 & 76
\end{array}\right) \\
& =\left(\begin{array}{cccc}
C & R & E & L \\
I & A & H & L \\
F & D & I & L
\end{array}\right)
\end{aligned}
$$

Portanto o destinatário encontrará a seguinte mensagem original: CIFRADEHILLL 


\section{Proposta de aula}

Nesta seção sugerimos uma proposta de aula que pode ser aplicada no Ensino Médio, no curso técnico em Informática, no curso de licenciatura em matemática ou em qualquer outro curso em que são ministrados os conteúdos de matrizes e programação de computadores. Esta proposta é baseada na aprendizagem significativa de David Ausubel. Nesta concepção, a aprendizagem se caracteriza pela interação entre os conhecimentos prévios e os conhecimentos novos, dando ao sujeito novos significados para os conhecimentos prévios.

Conforme Moreira [5], este conhecimento prévio pode ser um símbolo já significativo, um conceito, uma proposição, um modelo mental ou uma imagem chamado de subsunçor. Este é um "conhecimento específico, existente na estrutura do indivíduo, que permite dar significados a um novo conhecimento que lhe é apresentado ou por ele descoberto" [5].

O autor aponta que o conhecimento prévio é a variável que mais influencia a aprendizagem significativa de novos conhecimentos. Neste estudo, apontamos como o subsunçor os conhecimentos de lógica e programação de computadores, pois avaliamos como fundamentais para execução e compreensão desta proposta e são esses conhecimentos que darão "significados" ao conceito de matrizes.

Proposta de atividade: Construir um algoritmo para codificar e decodificar mensagens através do método de Hill.

Público-alvo: Alunos do Ensino Médio, do curso técnico em Informática ou do curso de licenciatura em matemática

Material necessário: Essa proposta de aula deve ser aplicada com o auxílio do laboratório de informática e, preferencialmente, com a participação dos professores de informática da escola. Será necessário quadro branco, pincel, retroprojetor, e ainda sugerimos que os computadores do laboratório de informática tenham a linguagem de programação C++ ou PHP.

Pré-Requisitos: Inicialmente o professor de matemática deve desenvolver com esses alunos os seguintes conteúdos matemáticos: matrizes, determinante, MDC e noções de aritmética modular.

\section{Objetivos:}

- Despertar o interesse dos alunos para o aprendizado da matemática.

- Associar teoria e prática matemática.

- Desenvolver a integração das disciplinas de matemática e programação de computadores.

- Aprender a trabalhar em equipe.

- Mostrar aplicações de conteúdos matemáticos no dia-a-dia.

Metodologia: Após o desenvolvimento dos conteúdos listados acima em pré-requisitos por meio de aula expositiva e dialogada, será explicado para os alunos o histórico da criptografia e o método aplicável em sala de aula, a cifra de Hill. Após o ensino dos conteúdos teóricos necessários, o professor pedirá que o alunos formem grupos de 5 membros e construam um 
algoritmo, em qualquer linguagem de programação, para codificar e decodificar uma mensagem utilizando o método de Hill.

Carga-horária prevista: Segue abaixo um cronograma com o tempo estimado de aula e o conteúdo a ser trabalhado. O tempo de uso do laboratório de informática fica a critério do professor envolvido com essa proposta de aula.

Tabela 3. Sugestão de cronograma para a proposta de aula

\begin{tabular}{cc}
\hline Tempo estimado & Conteúdo \\
\hline $4 \mathrm{~h} / \mathrm{a}$ & Divisão euclidiana, MDC, noções de aritmética modular. \\
\hline $1 \mathrm{~h} / \mathrm{a}$ & Números inversíveis em $\mathbb{Z}_{k}$. \\
\hline $1 \mathrm{~h} / \mathrm{a}$ & Matrizes: exemplos e operações. \\
\hline $4 \mathrm{~h} / \mathrm{a}$ & Determinante e Matriz Inversa. \\
\hline $3 \mathrm{~h} / \mathrm{a}$ & Criptografia e Cifra de Hill. \\
\hline
\end{tabular}

\section{Aplicação e resultados}

Nos meses de outubro e novembro de 2014, essa proposta de aula foi aplicada para os alunos do $3^{0}$ ano do curso técnico em informática do ensino integrado do Instituto Federal do Norte de Minas Gerais-IFNMG Campus: Arinos. No primeiro momento, os alunos assustaram-se quando ouviram as palavras algoritmo e programação dentro de um trabalho de matemática. A disciplina de programação de computadores foi vista somente no $1^{0}$ ano, ou seja, dois anos antes. Apesar disso, muitos alunos mostraram um grande interesse em participar do trabalho, já que cada integrante do grupo seria contemplado com 10 pontos dos 35 pontos distribuídos naquela etapa.

O primeiro passo foi explicar os conteúdos teóricos necessários. Noções de aritmética modular e números inversíveis foram novidade para os alunos, pois os mesmos nunca tinham visto esses temas. Os alunos mostraram grande dificuldade em efetuar divisões usando o algoritmo de divisão euclidiana. Uma situação que pode justificar essa dificuldade, é o fato dos alunos estarem acostumados a efetuar operações básicas de matemática em aparelhos eletrônicos. No conteúdo matrizes e determinantes, os alunos não tiveram muita dificuldade, pois muitos deles já tinham estudado o conteúdo no $2^{0}$ ano do curso. Quando foram citados os métodos de criptografia existentes e a importância da criptografia no contexto atual, foi o momento que os alunos mais prestaram atenção na aula, pois naquele momento eles estavam estudando conteúdos matemáticos associados à uma aplicação. Nesse momento percebe-se a importância de mostrar conteúdos matemáticos associados com fatos decorrentes do diaa-dia.

O segundo passo foi dividir a turma em grupos de 5 membros, aleatoriamente, para que os alunos pudessem ter interação entre eles. Em seguida, foi agendado o laboratório de informática para que os alunos pudessem todos os dias após as aulas regulares, se reunirem e confeccionarem o trabalho proposto. Os alunos mostraram grande dificuldade em traduzir as expressões matemáticas para a linguagem em que o seu algoritmo estava sendo construído. Nesse momento, a presença do professor de matemática é fundamental. As dificuldades de programação que iam surgido eram encaminhadas aos professores de informática, que participaram desses encontros que os alunos realizaram durante uma semana no laboratório. 
Revista Ciências Exatas e Naturais, Vol.19, nº.2, Jul/Dez, 2017

O terceiro passo, após terem criado o algoritmo de codificação pela cifra de Hill, foi apresentar o trabalho em sala de aula. Os alunos criaram uma interface, entregaram os códigos dos trabalhos feitos na linguagem $\mathrm{C}++$ e PHP, e ainda hospedaram o mesmo no servidor. Ao final da apresentação dos trabalhos, alguns alunos julgaram o algoritmo como trabalhoso, mas afirmaram que puderam relembrar códigos de programação e ainda aprenderam matemática.

Neste trabalho, os alunos utilizaram a tabela 4 abaixo, dos caracteres minúsculos (ao invés dos maiúsculos como fizemos na seção anterior) para fazerem a conversão das letras em números e ainda limitaram o tamanho da mensagem para apenas 9 caracteres.

Tabela 4. Conversão das letras minúsculas em sistema decimal sugerida pelos alunos

\begin{tabular}{ccccccccccccc}
\hline $\mathrm{a}$ & $\mathrm{b}$ & $\mathrm{c}$ & $\mathrm{d}$ & $\mathrm{e}$ & $\mathrm{f}$ & $\mathrm{g}$ & $\mathrm{h}$ & $\mathrm{i}$ & $\mathrm{j}$ & $\mathrm{k}$ & $\mathrm{l}$ & $\mathrm{m}$ \\
\hline 0 & 1 & 2 & 3 & 4 & 5 & 6 & 7 & 8 & 9 & 10 & 11 & 12 \\
\hline $\mathrm{n}$ & $\mathrm{o}$ & $\mathrm{p}$ & $\mathrm{q}$ & $\mathrm{r}$ & $\mathrm{s}$ & $\mathrm{t}$ & $\mathrm{u}$ & $\mathrm{v}$ & $\mathrm{w}$ & $\mathrm{x}$ & $\mathrm{y}$ & $\mathrm{z}$ \\
\hline 13 & 14 & 15 & 16 & 17 & 18 & 19 & 20 & 21 & 22 & 23 & 24 & 25 \\
\hline
\end{tabular}

\section{Conclusão}

Existem várias tópicos da matemática que podem ser trabalhados com aplicações como o que foi proposto acima. Isso ajudaria a responder a pergunta clássica de uma aula de matemática: "Para que isso servirá em minha vida?". O algoritmo construído pelos alunos teve um aspecto positivo, pois, contribuiu para o desenvolvimento dos alunos no estudo de matrizes e na programação, auxiliando o aprendizado dessas disciplinas. Esse trabalho não esgotará o estudo sobre aplicações na matemática, espera-se que a partir dele possam surgir novas ideias para associar a teoria à prática em sala de aula, podendo assim melhorar o aprendizado em matemática.

\section{Referências}

[1] D’Ambrosio, B. S. Como Ensinar Matemática Hoje? SBEM, Brasília, ano 2, n.2, p.15-19, 1989.

[2] D'AMBROSIO, Ubiratan. Ação pedagógica e Etnomatemática como marcos conceituais de Matemática. In: BICUDO, M.A.V.; Educação Matemática. Editora Centauro. $2^{a}$ ed. 2005 .

[3] BRASIL, Ministério da Educação, Parâmetros Curriculares Nacionais: ensino médio. Secretaria de Educação Média e Tecnológica. Brasília: 1999.

[4] CHARLOT, B. Da relação com o saber às práticas educativas. São Paulo: Cortez, 2013.

[5] MOREIRA, M. A. Aprendizagem significativa: a teoria e textos complementares. São Paulo: Editora Livraria de Física, 2011. 
BARBOSA, L. D. A. e CORNELISSEN, M. G.

[6] SCHURMANN, H. Criptografia matricial aplicada ao ensino médio. 2013. 75 f. Dissertação (Mestrado Profissional em Matemática) - Universidade Estadual de Londrina, Centro de Ciências Exatas, Programa de Pós-Graduação em Matemática, 2013.

[7] MARINHO FILHO, E. R. Criptografia: uma engenharia didática com funções matrizes e cifra de Hill, para o ensino médio. 2015. 128 f. Dissertação (Mestrado Profissional em Matemática) - Universidade Federal do Oeste do Pará, Instituto de Ciências da Educação, Programa de Ciências Exatas.

[8] OLIVEIRA, W. F. Uma proposta para ampliar a perspectiva de professores e alunos em relação ao estudo se matrizes. 2017. 129 f.Dissertação (Mestrado Profissional em Matemática) - Universidade Estadual Paulista "Júlio de Mesquita Filho", Instituto de Biociências, Letras e Ciências Exatas.

[9] CONCEIÇÃO, M. R. F. Transformações no Plano: Uma Aplicação do Estudo de Matrizes com o Uso de Planilhas Eletrônicas. 2013. 64 f. Dissertação (Mestrado Profissional em Matemática em Rede Nacional - PROFMAT). Instituto de Matemática, Estatística e Física da Universidade Federal do Rio Grande, 2013.

[10] FALEIROS, A. C. Criptografia. São Carlos-SP: SBMAC,2011.

[11] FIARRESGA, V. M. C. Criptografia e Matemática. (Dissertação do Mestrado em Matemática para Professores). Departamento de Matemática, Universidade Federal de Lisboa, 2010 .

[12] EVARISTO, J.; PERDIGÂO, E. Introdução à Álgebra Abstrata. Maceió: EDUFAL,2002.

[13] COUTINHO, S.C. Números Inteiros e Criptografia RSA. Rio de Janeiro: IMPA, 2014.

[14] SEYMOUR, L. Álgebra Linear: teorema e problemas.São Paulo: Pearson Makron Books, 1994.-(Coleção Schaum)

[15] HEFEZ, A. Aritmética.Rio de Janeiro: SBM,2013.

[16] DOMINGUES, H. H.;IEZZI, G. Álgebra Moderna.São Paulo: Atual,2003.

[17] SHOKRANIAN, S. Uma introdução à Teoria dos números. Rio de Janeiro: Editora Ciência Moderna Ltda, 2008.

[18] HEFEZ, A.; FERNANDEZ, C. S. Introdução à Álgebra Linear. Rio de Janeiro: SBM,2012.

[19] LIMA, E. L. Álgebra linear.Rio de Janeiro:SBM,2003.

[20] RIBEnBOIM, P. Números Primos: Velhos Mistérios e Novos Recordes. Rio de Janeiro: IMPA, 2012.

[21] SANTOS, R. J. Introdução a Álgebra Linear. Departamento de MatemáticaICEx.Universidade Federal de Minas Gerais, 2010. 